\title{
Saliendo de la zona de comodidad
}

\author{
Edna Gabriela Delgado Quiñones
}

\section{Resumen}

En este relato describo los cambios que he pasado como docente, los obstáculos que he debido enfrentar para no perder las clases con mis alumnos, las preguntas y reflexiones que me he formulado durante este tiempo y, finalmente, las actividades que he desarrollado. Sin duda, esta contingencia ha sido una oportunidad para aprender, para que alumnos y docentes utilicemos la tecnología a nuestro favor, brinquemos obstáculos y, principalmente, desarrollemos habilidades propias como el autoconocimiento y la autorregulación, para llevar a cabo una metacognición.

Palabras clave: comodidad, motivación, enseñanza-aprendizaje, docencia.

\section{GetTING OUT OF THE COMFORT ZONE}

\begin{abstract}
In this text I describe the changes that I have gone through as a teacher, the obstacles that I have confronted not to lose classes with my students, the questions and reflexions that I have had during this time, and finally, the activities I have developed. Undoubtedly, this contingency has been a learning opportunity for students and teachers to use technology to our advantage, to overcome obstacles and, mainly, to develop our own abilities, such as self-knowledge and self-regulation, in order to carry out metacognition.
\end{abstract}

Keywords: comfort, motivation, teaching-learning process, teaching. 
"Saliendo de la zona de comodidad"

Edna Gabriela Delgado Quiñones

Vol. 22, Núm. 1, enero-febrero 2021

Revista Digital Universitaria

Dra. Edna Gabriela Delgado Quiñones

dra.ednagdq@hotmail.com

orcid.org/0000-0002-7734-6494

Medico Familiar, Maestra en Ciencias de la Educación y Procesos Cognitivos, adscrita al Instituto Mexicano del Seguro Social, Guadalajara, Jalisco. Con trayectoria en docencia e investigación clínica y educativa.

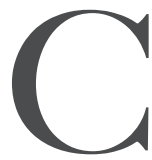

omo docente, he tenido un duelo con esta pandemia. Es decir, el cambio me obligó a salirme de mi zona de comodidad. Mis planes operativos, estrategias didácticas y evaluaciones cambiaron de un momento a otro. La educación digital se volvió una realidad, así que tuve que familiarizarme con las plataformas educativas y buscar en ellas la manera de atraer a mis alumnos.

Fui obligada a cambiar mis tradicionales presentaciones de Power Point por sesiones en Zoom. Esta "obligación" surgió desde mi vocación docente, desde el saber que mis alumnos perderían sus clases. Todo esto me motivó a estudiar, a investigar, a realizar ensayo y error, y a alcanzar nuevas estrategias educativas. Con el tiempo las fui valorando, conociendo mis aciertos y errores, para después cuestionarme: ¿realmente estoy logrando un aprendizaje significativo en mis alumnos?, ¿la forma de comunicarme vía sincrónica o asincrónica será asertiva?, ¿cómo puedo motivarlos a trabajar de esta manera, si ni yo entiendo? Cuando finalmente llegaron las evaluaciones, me surgieron aún más preguntas: ¿cómo evaluar a mis alumnos? y ¿cómo reconocer su esfuerzo, su presencia, su puntualidad y su intento por trabajar a la par? 
"Saliendo de la zona de comodidad" Edna Gabriela Delgado Quiñones

Nuestra preparación docente quedó en jaque. Si tenías ya conocimientos sobre las NTIC, ahora el aplicarlas era una realidad. Tuvimos que elaborar y construir en una plataforma, además de aprender a diversificar las técnicas y estudios. Esto nos permitió reflexionar, fortalecer nuestra vocación. Propició el instruirnos en cómo optimizar los tiempos, cómo fomentar un proceso que impacte en un aprendizaje significativo, y en ver la era digital como una realidad factible que debe ser utilizada como una gran herramienta de apoyo, aun cuando terminé esta situación de salud.

La pandemia nos ha ofrecido la oportunidad de lograr en nuestros alumnos un autoconocimiento y una autorregulación, para que al final tengan las herramientas necesarias para lograr una metacognición y que una escuela o instituto cerrado no sea un obstáculo para seguir aprendiendo. Así que, a pesar de todo, agradezco esta oportunidad de salir de mi zona de comodidad, de esforzarme para mejorar mi desempeño profesional, de lograr en mis alumnos una percepción diferente de la realidad y ser para ellos un ejemplo de perseverancia.

Y así como en el pasado se cambió el pizarrón verde con gis por diapositivas, actualmente se están cambiando las diapositivas por las NTIC y estamos aprendiendo a utilizar las redes sociales para fines educativos.

\section{Cómo CITAR ESTE ARTículo}

- Delgado Quiñones, Edna Gabriela. (2021, enero-febrero). Saliendo de la zona de comodidad. Revista Digital Universitaria (RDU), 22(1). Dol: http://doi.org/10.22201/ cuaieed.16076079e.2021.22.1.4 